\title{
The Course of Heart Failure Development and Mortality in Rats with Volume Overload due to Aorto-Caval Fistula
}

\author{
Vojtech Melenovsky ${ }^{a}$ Petra Skaroupkova ${ }^{b}$ Jan Benes ${ }^{a}$ Vera Torresova ${ }^{b}$ \\ Libor Kopkan $^{\text {b }}$ Ludek Cervenkac \\ ${ }^{a}$ Department of Cardiology and ${ }^{b}$ Center for Cardiovascular Research, Institute for Clinical and Experimental \\ Medicine - IKEM, Prague, and ' Department of Physiology, 2nd Faculty of Medicine, Charles University, Prague, \\ Czech Republic
}

\section{Key Words}

Heart failure $\cdot$ Volume overload $\cdot$ Mortality .

Cardiac hypertrophy $\cdot$ Experimental models

\begin{abstract}
Background: There are only few studies documenting the long-term outcome of aorto-caval fistula (ACF) in rats, a model of volume overload heart failure (HF). The aim of the present study was to describe HF-related morbidity and mortality, and to examine the relation between cardiac hypertrophy and survival. Methods: Adult male Wistar rats underwent needle ACF or sham operation and 71 animals surviving the acute procedure with patent ACF were followed for 52 weeks. Results: By the end of the study, $72 \%$ of the ACF animals deceased and $82 \%$ developed HF signs. Of the HF rats, $65 \%$ died (median: 3 weeks after HF onset). Before death, body weight increased by $9 \%$ followed by a final drop. $28 \%$ ACF rats died suddenly, without preceding HF. Sudden death occurred earlier and in the rats with a trend to larger hearts $(p=0.07)$. In the whole ACF cohort, heart weight (heart weight/body weight ratio) was inversely associated with the length of survival $(r=-0.51, p<0.001)$. Conclusion:
\end{abstract}

The median survival of ACF Wistar rats is 43 weeks, longer than reported in other rat strains. Increased heart weight is associated with higher mortality and a significant number of animals die suddenly.

Copyright $\odot 2011$ S. Karger AG, Basel

\section{Introduction}

Chronic volume overload due to aorto-caval fistula (ACF) in rats is one of several well-characterized rodent models of heart failure (HF) [1-5]. The model is simple, reproducible and it has several key features of human HF, such as the gradual transition from the asymptomatic into the decompensated phase, marked neurohumoral activation [1], fluid retention and changes in myocardial phenotype typical of HF [6]. The creation of an artificial shunt between the abdominal aorta and the inferior vena cava with a needle [2] causes a marked increase in venous return and cardiac output that triggers compensatory, initially asymptomatic ventricular hypertrophy [7]. Due to persistent hemodynamic overload, redistribution of cardiac output and activation of neurohumoral response,

\section{KARGER}

Fax +4161306 1234 E-Mail karger@karger.ch www.karger.com
Vojtech Melenovsky, MD, $\mathrm{PhD}$

Department of Cardiology

Institute for Clinical and Experimental Medicine - IKEM

Videnska 1958/9, CZ-14021 Prague 4 (Czech Republic)

Tel. +420 236055 190, E-Mail vojtech.melenovsky@ikem.cz 
HF starts to develop 8-10 weeks after ACF induction. Overt $\mathrm{HF}$ with venous congestion is present in the majority of rats after 20-30 weeks, at least in the Sprague-Dawley rat strain [4]. There are only few studies documenting the long-term consequences of ACF, although this model would be suitable for the testing of interventions that target the advanced stage of HF. Notably, an appropriate animal HF model is crucial for the development of new pharmacological therapies, as it was in the case of angiotensin-converting enzyme inhibitors [8]. The model has also been particularly useful for studies examining renal manifestations of HF and the balance between sodiumretaining and natriuretic systems in the kidney $[9,10]$.

The primary aim of the present study was to describe the course of HF-related morbidity and mortality in male Wistar rats with chronic volume overload induced by ACF. The secondary aim was to examine the relation between the extent of cardiac hypertrophy and the length of survival that has never been directly addressed in this model.

\section{Methods}

HF was induced by volume overload from ACF created using the needle technique $[2,4,11]$. Male Wistar rats (AnLab Ltd, Prague, Czech Republic) weighing 300-350 g were anesthetized with a ketamine/midazolam mixture and a midline abdominal laparotomy was performed to expose the infrarenal aorta and the vena cava. An 18-gauge needle (diameter $1.2 \mathrm{~mm}$; Becton-Dickinson) was inserted into the abdominal aorta and advanced through the medial wall into the vena cava to create ACF. The aorta above the place of puncture was then temporarily clamped, the needle withdrawn and the puncture site was sealed with cyanoacrylate glue. The creation of ACF was confirmed by observing pulsatile, bright flow in the inferior vena cava. The abdominal cavity was closed by an absorbable suture. In the control (sham) group, animals underwent the identical procedure, but without the puncture of the vessels. The animals were kept on a $12 / 12$ hour light/dark cycle and were fed normal salt/protein diet $(0.45 \%$ $\mathrm{NaCl}, 19-21 \%$ protein; Semed Ltd, Prague, Czech Republic). The animals were regularly inspected and weighed weekly by a single experienced technician who also monitored for presence of $\mathrm{HF}$ symptoms, similarly as done previously in a rat SHHF model [12, 13]. To better quantify HF symptoms, every animal was scored $0-3$ points $(0=$ absence, $3=$ maximal presence $)$ in five most apparent aspects of the rat HF phenotype: (1) presence of raised fur (piloerection), (2) diminished activity (lethargy), (3) peripheral cyanosis, (4) rapid or labored breathing (dyspnea), and (5) abdominal swelling (ascites). A weekly heart failure score was calculated for every animal as the sum of individual points and the animals were classified as having HF once they have ever reached a mandatory HF score threshold $>3$. The advantage of this scoring system is that it allows repeated non-invasive assessment. The animals that died during the experiment underwent autopsy within
$24 \mathrm{~h}$ of death, the hearts were removed, clotted blood was removed and the hearts were weighed. The results were analyzed using a Statisica software package. The groups were compared using an unpaired $t$ test and the results are expressed as means \pm SD. The investigation conformed to the NIH Guide for the Care and Use of Laboratory Animals (NIH Publ. No. 85-23, 1996), the Animal Protection Law of the Czech Republic (311/1997) and was approved by the Ethics Committee of IKEM.

\section{Results}

The ACF procedure was associated with $13 \%$ early $(\leq 7$ days) mortality, occurring mostly within the first $48 \mathrm{~h}$, and these animals were excluded from all analyses. Eighty-two animals survived the perioperative period and entered the study. At the end of the study, the ACF animals with normal heart weights $[\mathrm{n}=11$, heart weight/ body weight $(\mathrm{HW} / \mathrm{BW})<$ mean $+2 \times \mathrm{SD}$ of $\mathrm{HF} / \mathrm{BW}$ of sham-operated group] were further excluded from all analyses because of probable loss of fistula patency. The remaining 71 animals were analyzed and reported.

\section{Morbidity and Mortality}

In the sham-operated group, no animal died before the end of the study. In the ACF group, the first death occurred in the 18th week. By the end of the study (52nd week), $72 \%$ of ACF animals were dead (51/71), with a median survival of 43 weeks post-ACF procedure (fig. 1c). Over the course of the study, 82\% (58/71) of ACF animals crossed the HF threshold (ever recorded HF score sum $>3$; fig. 1d), and $65 \%$ of them (38/58) died. Median HFfree survival was 40 weeks post-ACF procedure. Only $72 \%$ of animals that died had HF (HF death), the rest of ACF animals (28\%) died suddenly, without crossing the threshold for HF. Starting from approximately the 20th week, the HF score in the ACF group started to rise almost linearly with time (fig. 2). At the last examination before death or study end, the most frequently observed abnormality ( $\geq 1$ grade) in ACF animals was piloerection (observed in $83 \%$ ), followed by abdominal swelling (79\%), labored breathing (34\%), cyanosis (10\%) and lethargy (4.2\%). Most of non-HF deaths occurred early (fig. 3b), but after the 30th week, HF-onset preceded mortality (fig. 1c, d). The interval between HF onset and death (in lethal $\mathrm{HF}, \mathrm{n}=38$ ) has a left-skewed distribution with a median of 3 weeks (interquartile range: 6 ). The interval between HF onset and the end of the follow-up (in nonlethal HF, $\mathrm{n}=20$ ) had a normal distribution with a mean of 10.5 weeks (SD: 4.6). 


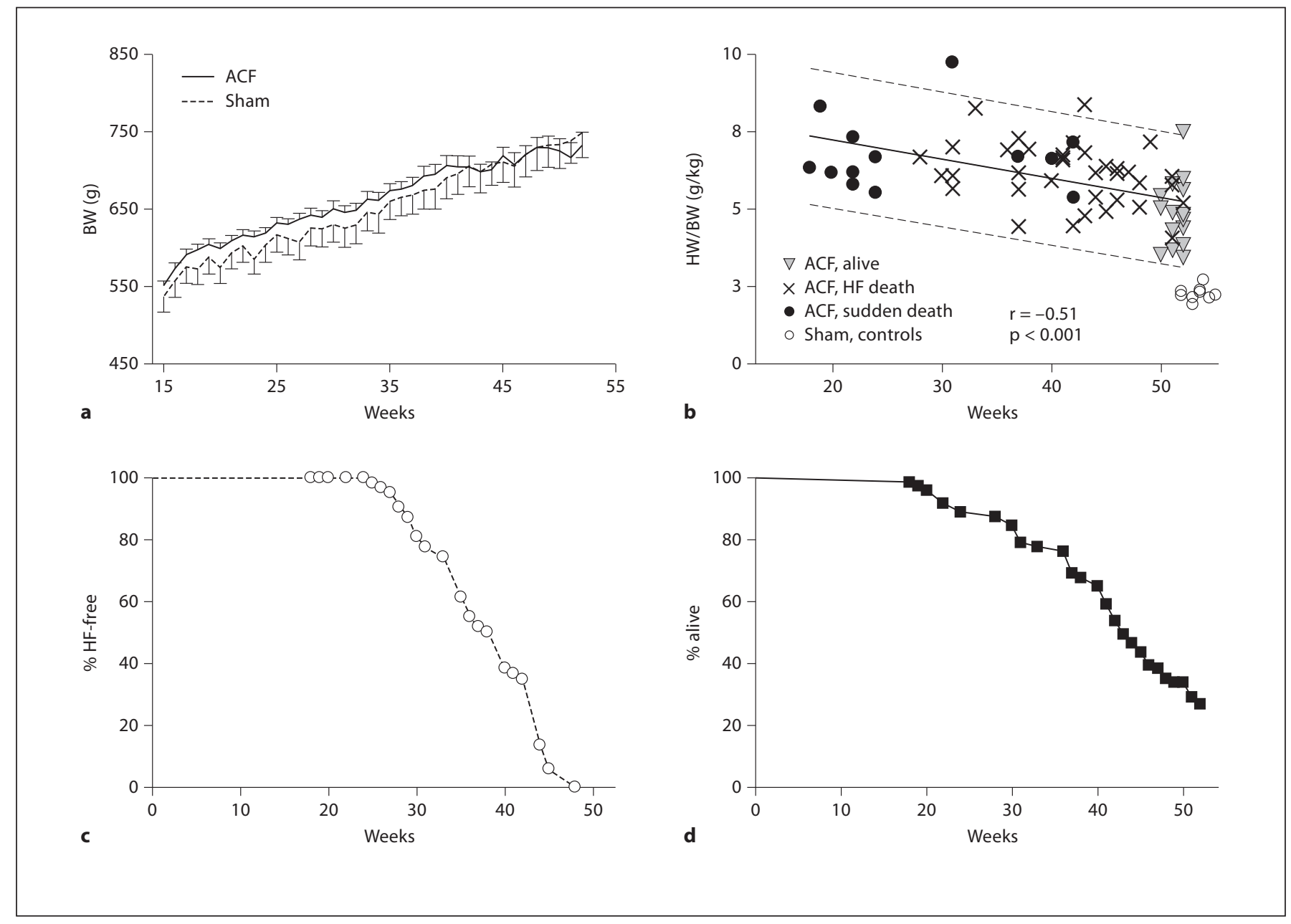

Fig. 1. a Changes in BW in sham-operated animals and in animals with ACF. b The relation of HW (normalized for BW, HW/BW) to survival in all animals with ACF. c Kaplan-Meier graph depicting time to onset of HF in ACF animals (defined by each recorded HF score sum > 3). d Kaplan-Meier graph depicting time to death in ACF animals.

\section{$B W$ Changes before Death}

Averaged BW curves were similar in ACF and shamoperated animals (fig. 1a). However, deceased animals displayed a biphasic pattern of BW change before death (fig. 3b) when compared to BW of the sham-operated group. BW (expressed as \% of BW of controls) in the ACF group started to increase 4 weeks before death, reached a maximum of $9 \%(\mathrm{p}=0.005)$ on the week -1 and then dropped, thus BW at death was not significantly different from the sham-operated group. This pattern probably reflects an early increase in congestion and then later anorexia. The profile of BW changes before death was similar between animals with HF and non-HF death. The absolute magnitude of weight changes was smaller in the
non-HF group, probably due to a higher proportion of younger animals with lower BW.

\section{Relation between Cardiac Hypertrophy and Survival}

In animals with ACF, BW-adjusted HW was inversely associated with the length of survival $(\mathrm{r}=-0.51, \mathrm{p}<0.001$; fig. 1b). The relation of BW-unadjusted HW to survival went in a similar direction, although weaker $(r=-0.33$, $\mathrm{p}=0.005)$. ACF animals that died during the study had a higher HW/BW index ( $<<0.01$, by $30 \%)$ than surviving ACF animals (table 1; fig. 1b). ACF animals dying suddenly without previous HF signs $(\mathrm{HF}$ score $<3$ ) tended to have a higher HW/BW index (by $10 \%, \mathrm{p}=0.07$ ) than animals dying from progressive HF (table 1). 
Fig. 2. Averaged weekly HF score sum and its components (scale $0-3$ ) obtained from every surviving ACF animal.

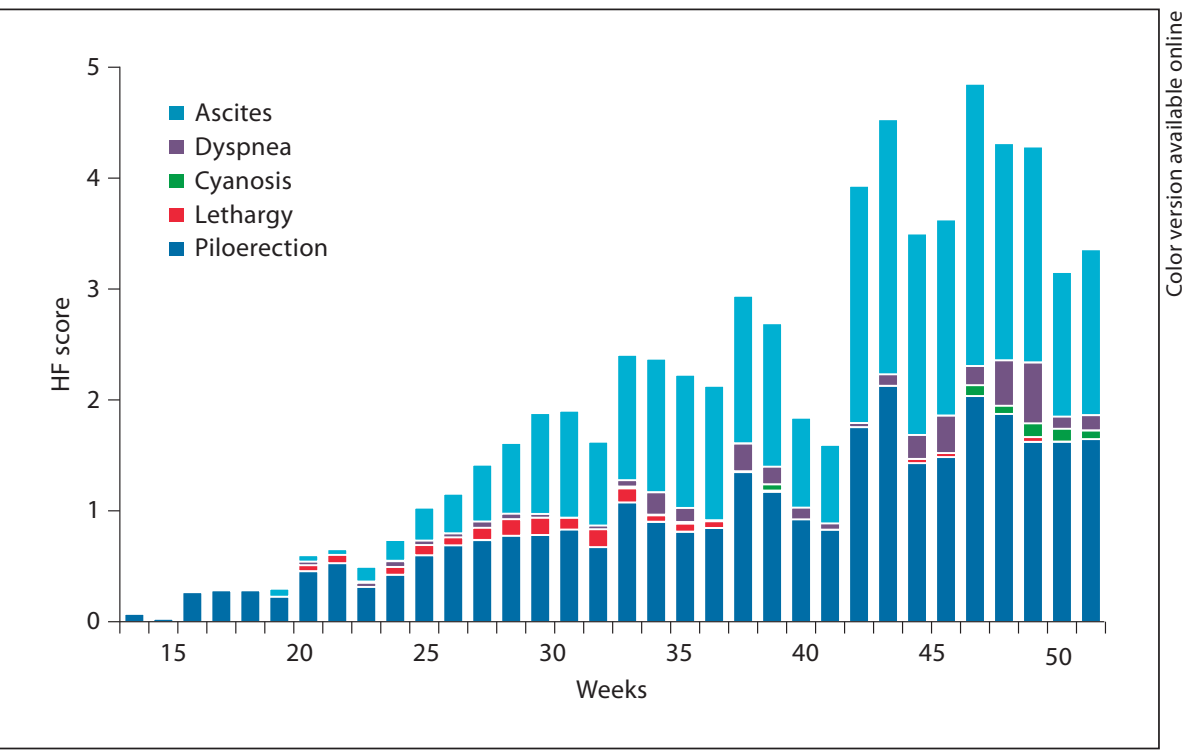

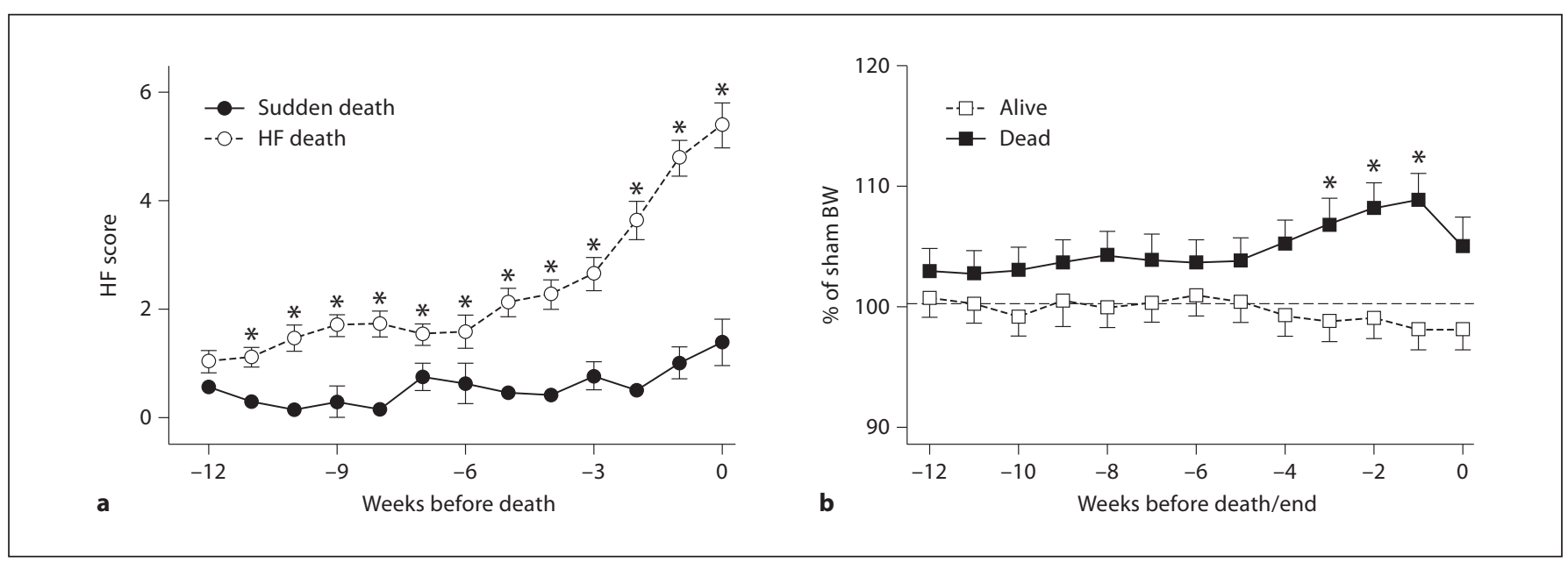

Fig. 3. a Sum of HF score before death in animals dying from HF and animals dying without previous signs of HF. b Ratio of BW in ACF animals over BW of control (sham-operated) rats before death or before the end of the study. ${ }^{*} \mathrm{p}<0.05$ vs. animals with sudden death.

\section{Discussion}

The present study found that $82 \%$ of animals with ACF developed HF and $72 \%$ died during the 1-year study period. The majority of HF animals died soon after the onset of HF signs (with a median of 3 weeks), but $38 \%$ of them survived in a HF state till the end of the study, indicating that the onset of HF is not a reliable surrogate for mortality. Survival in our study is longer than previously reported in a study where male Sprague-Dawley rats with ACF demonstrated $\geq 80 \%$ HF-related morbidity and mortality as soon as 20 weeks after ACF formation [14]. This discrepancy can be explained by the fact that in the former report, authors did not measure the time to actual death, but rather the time to combined endpoint of death or HF onset (defined there by an arbitrary weight increment of $50 \mathrm{~g}$ plus other signs of HF). An alternative explanation could be related to the differences between the rat strains, because Sprague-Dawley rats might be more prone to HF development than Wistar rats [15]. Our observation of a relatively long survival in the ACF model is in line with a recent report showing that volume 
Table 1. HF status and survival by the end of the study

\begin{tabular}{|c|c|c|c|c|c|c|}
\hline & \multirow[t]{2}{*}{ Sham } & \multirow[t]{2}{*}{$\mathrm{ACF}$} & \multicolumn{2}{|l|}{ All ACF } & \multicolumn{2}{|c|}{ All dead ACF } \\
\hline & & & alive & dead & HF death & sudden death \\
\hline Number & 10 & 71 & 20 & 51 & 38 & 13 \\
\hline Alive, $\mathrm{n}$ & $100 \%$ & $20(28 \%)$ & $100 \%$ & $0 \%$ & $0 \%$ & $0 \%$ \\
\hline HF present ${ }^{1}, \mathrm{n}$ & - & $8(58 \%)$ & $20(100 \%)$ & $38(74 \%)$ & $100 \%$ & $0 \%$ \\
\hline Follow-up, weeks & $52 \pm 0.3$ & $41.7 \pm 10^{*}$ & $51.5 \pm 0.8$ & $37.8 \pm 9.3$ & $41.2 \pm 6.6$ & $27.9 \pm 9.1^{*}$ \\
\hline Final body weight ${ }^{2}, g$ & $745 \pm 94$ & $682 \pm 103$ & $725 \pm 54$ & $665 \pm 113^{*}$ & $678 \pm 122$ & $625 \pm 70$ \\
\hline Heart weight, $g$ & $1.67 \pm 0.2$ & $3.97 \pm 0.8^{*}$ & $3.50 \pm 0.6$ & $4.15 \pm 0.8^{*}$ & $4.15 \pm 0.8$ & $4.18 \pm 0.6$ \\
\hline $\mathrm{HW} / \mathrm{BW}$ ratio, g/kg & $2.25 \pm 0.2$ & $5.89 \pm 1.2^{*}$ & $4.84 \pm 1.0$ & $6.29 \pm 1.0^{*}$ & $6.14 \pm 1.0$ & $6.75 \pm 1.2^{* *}$ \\
\hline HF score sum & - & $4.01 \pm 2.6^{*}$ & $3.15 \pm 1.3$ & $4.35 \pm 2.9$ & $5.50 \pm 2.4$ & $1.00 \pm 1.3^{*}$ \\
\hline Piloerection & - & $1.69 \pm 1.0^{*}$ & $1.60 \pm 0.6$ & $1.73 \pm 1.1$ & $2.14 \pm 0.9$ & $0.5 \pm 0.8^{*}$ \\
\hline Lethargy & - & $0.04 \pm 0.2$ & $0.01 \pm 0$ & $0.06 \pm 0.2$ & $0.08 \pm 0.3$ & $0.0 \pm 0.0$ \\
\hline Cyanosis & - & $0.10 \pm 0.3$ & $0.10 \pm 0.3$ & $0.10 \pm 0.3$ & $0.13 \pm 0.3$ & $0.0 \pm 0.0$ \\
\hline Dyspnea & - & $0.52 \pm 0.8$ & $0.05 \pm 0.2$ & $0.71 \pm 0.4^{*}$ & $0.92 \pm 0.9$ & $0.08 \pm 0.3^{*}$ \\
\hline Ascites & - & $1.65 \pm 1.1^{*}$ & $1.40 \pm 0.9$ & $1.75 \pm 1.1$ & $2.22 \pm 0.9$ & $0.38 \pm 0.6^{*}$ \\
\hline
\end{tabular}

${ }^{*} \mathrm{p}<0.05,{ }^{* *} \mathrm{p}=0.07 .{ }^{1}$ Defined as ever attained HF score $>3 .{ }^{2}$ Before death or study end.

overload has a lower mortality than pressure overload at the same level of mean left ventricular wall stress [6].

Interestingly, as many as $28 \%$ of the ACF animals died suddenly, without preexisting signs of HF. These animals had marginally larger hearts and the death occurred earlier after ACF induction than in animals with preexisting HF signs. The reasons for a high incidence of sudden death in ACF animals are unknown, but the most likely possibility are malignant ventricular arrhythmias, common also in humans with cardiac hypertrophy, due to volume overload [16]. The arrhythmic substrate for malignant ventricular arrhythmias in the ACF model may not be myocardial fibrosis, but rather a gap junction remodeling [17]. An alternative reason for sudden death could be a rapidly progressive cardiac decompensation that might have been missed on weekly HF scoring.

We also attempted to address the unresolved relation between cardiac hypertrophy and survival in chronic volume overload. We found a significant inverse correlation $(\mathrm{r}=-0.51, \mathrm{p}<0.001)$ between these two parameters, i.e. ACF animals with relatively larger hearts survived for a shorter time than ACF animals with smaller hearts. In contrast, Brower et al. [3, 4] found no straightforward correlation between the degree of cardiac hypertrophy and the development of HF or death; this was supported by the lack of difference in left ventricular mass between animals with or without $\mathrm{HF}$, although the failing ventricles had larger diastolic diameters. These findings al- lowed authors to speculate that greater cardiac hypertrophy is protective in ACF and the survival is shorter in animals with smaller hearts due to insufficient hypertrophy'. Our study contradicts this observation and indicates that increased HW is associated with shortened survival, similarly to the situation for example in pressure overload-induced hypertrophy [18]. The discrepant findings with regard to our study could be explained by imprecise determination of actual survival in the former study, where a combined endpoint of death or presence of HF was used for 'survival' analysis. Our finding is in line with the known positive association between cardiac hypertrophy and the occurrence of HF or death that has been demonstrated in the general human population [19]. However, from our data, we cannot address the possible role of cardiac chamber dilatation or relative wall thinning that may both have effects on the onset of HF, independently of the hypertrophic response [20]. The important question that should be addressed in future studies is whether the inhibition of such hypertrophic response by drugs or genomic manipulations, at the same degree of prevailing hemodynamic overload from ACF, would delay the development of HF.

For the definition and monitoring of HF progression, we used our own HF score that quantifies the appearance of rats with $\mathrm{HF}[12,13]$ and does not rely only on arbitrary weight change. These signs are not entirely specific to HF and might also reflect environmental influences, but 
when these signs are consecutively acquired in an entire animal cohort with preexisting cardiac pathology, they are likely to capture the onset of HF. The most often observed abnormality was piloerection (raised fur) that might reflect sympathetic activation and/or decreased tolerance of the effort that rat needs for fur hygiene. In contrast, we did not observe fluid accumulation in the extremities. The probable reason is that fluid retentionpromoting effects of gravity on blood column in leg veins is likely minimal in mostly recumbent animals with short legs. Importantly, we noticed that the BW changes in ACF animals before death were relatively small, occurred later than the changes in HF score and showed a biphasic trend, with an initial increase of BW (+8\%) starting 3-4 weeks prior to death, followed by a preterminal decrease of BW. Thus, the effects of fluid retention on BW in ACF might be partly offset by cachexia, changes in body composition and anorexia. This might be a source of error in HF diagnosis based primarily on the weight change.

Our study has several limitations. First, we analyzed only male rats and there are known gender differences in the cardiac adaptation to volume overload. However, the impact of ACF on cardiac function and geometry is larger in males than in females [21]. We measured the weights of the whole heart, but not of the individual chambers. We believe that this is adequate, since volume overload is imposed on all four cardiac chambers, and not just on the left ventricle. On the other hand, we acknowledge that the time course of hypertrophic response to imposed stress might differ due to intrinsic developmental differences between right and left ventricle [22, 23]. The measured HW was normalized to BW and not to a tibial length. From previous studies $[17,24,25]$ we know that presence of ACF has no major effect on the BW-tibial length relation. Likewise, BWs at the time of death were rather similar to the sham group (fig. 3b), so an alternative denominator for HW normalization would likely provide similar results. We cannot address the source of the HW variance and we were not able to separate possible effects of a minute variation in the shunt size from an intrinsic variation of the hypertrophic response. However, the rats with normal HW (defined as $<2$ SD from mean of the sham group) that often have closed ACF, were excluded from all analyses. We fully acknowledge that the HF score is based on rather unspecific factors, but it represents an analogy to clinical probabilistic approach: HF diagnosis in humans is based on a presence of cardiac abnormality and presence of (quite unspecific) signs and symptoms [26], rather than on a single, unique parameter. The relation of HF development to cardiac structure and function was not examined using echocardiography and deserves further studies.

In summary, we describe here the course of HF-related morbidity and mortality in ACF-induced volume overload in male Wistar rats. The time to the HF onset and survival (median: 43 weeks) was longer than reported previously. As many as $28 \%$ of the animals died suddenly, without previous HF signs, probably due to arrhythmiarelated death. In the whole cohort, increased HWs were associated with shorter survival, indicating that an excessive hypertrophy in the ACF model predisposes to the development of HF or death, similarly as in other models of hemodynamic overload. The study provides novel data about the long-term trajectory of HF development and provides a foundation for planning of interventional studies using this experimental model of advanced HF.

\section{Acknowledgements}

This work was supported by the EU Operational Program Prague - Competitiveness, project 'CEVKOON' (CZ.2.16/ 3.1.00/22126 to L.C.), by the Ministry of Health (MZO-00023001 to V.M. and L.C., NS10497-3/2009 to V.M.), the Ministry of Education (MSMT-1MO510 to V.M., J.B. and L.C.) and by the Grant Agency of the Czech Republic (305/09/1390 to V.M.).

References

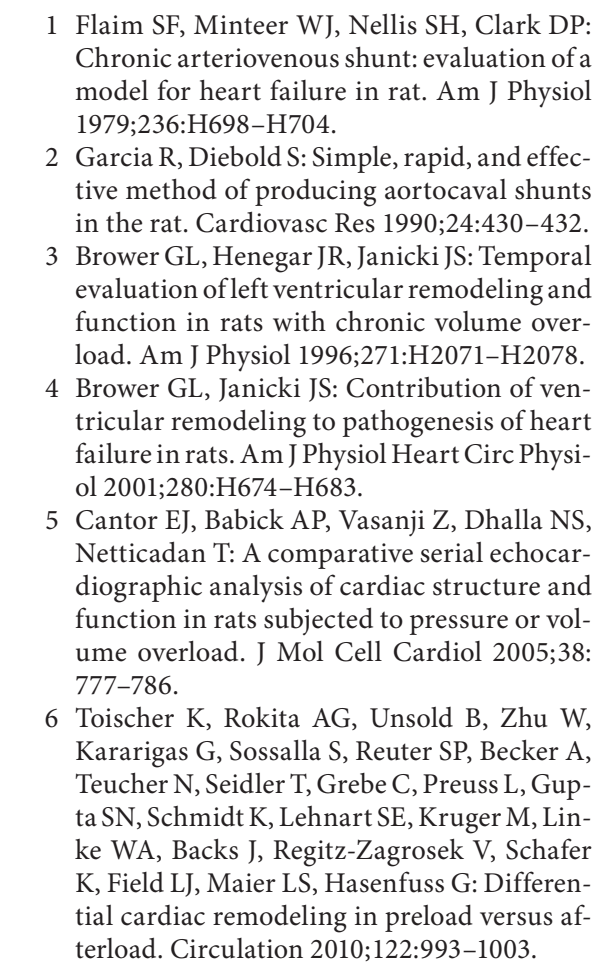

Melenovsky/Skaroupkova/Benes/ Torresova/Kopkan/Cervenka 
7 Ruzicka M, Yuan B, Leenen FH: Effects of enalapril versus losartan on regression of volume overload-induced cardiac hypertrophy in rats. Circulation 1994;90:484-491.

-8 Pfeffer MA, Pfeffer JM, Steinberg C, Finn P: Survival after an experimental myocardial infarction: beneficial effects of long-term therapy with captopril. Circulation 1985;72: 406-412.

-9 Abassi ZA, Brodsky S, Karram T, Dobkin I, Winaver J, Hoffman A: Temporal changes in natriuretic and antinatriuretic systems after closure of a large arteriovenous fistula. Cardiovasc Res 2001;51:567-576.

10 Willenbrock R, Pagel I, Scheuermann M, Hohnel K, Mackenzie HS, Brenner BM, Dietz R: Renal function in high-output heart failure in rats: role of endogenous natriuretic peptides. J Am Soc Nephrol 1999;10:572580.

-11 Ruzicka M, Yuan B, Harmsen E, Leenen FH: The renin-angiotensin system and volume overload-induced cardiac hypertrophy in rats. Effects of angiotensin converting enzyme inhibitor versus angiotensin II receptor blocker. Circulation 1993;87:921-930.

$\checkmark 12$ Emter CA, McCune SA, Sparagna GC, Radin MJ, Moore RL: Low-intensity exercise training delays onset of decompensated heart failure in spontaneously hypertensive heart failure rats. Am J Physiol Heart Circ Physiol 2005;289:H2030-H2038.

-13 Chicco AJ, McCune SA, Emter CA, Sparagna GC, Rees ML, Bolden DA, Marshall KD, Murphy RC, Moore RL: Low-intensity exercise training delays heart failure and improves survival in female hypertensive heart failure rats. Hypertension 2008;51:10961102.
14 Janicki JS, Brower GL, Henegar JR, Wang L: Ventricular remodeling in heart failure: the role of myocardial collagen. Adv Exp Med Biol 1995;382:239-245.

15 Oliver-Dussault C, Ascah A, Marcil M, Matas J, Picard S, Pibarot P, Burelle Y, Deschepper CF: Early predictors of cardiac decompensation in experimental volume overload. Mol Cell Biochem 2010;338:271-282.

16 Grigioni F, Enriquez-Sarano M, Ling LH, Bailey KR, Seward JB, Tajik AJ, Frye RL: Sudden death in mitral regurgitation due to flail leaflet. J Am Coll Cardiol 1999;34:20782085.

17 Benes J Jr, Melenovsky V, Skaroupkova P, Pospisilova J, Petrak J, Cervenka L, Sedmera D: Myocardial morphological characteristics and proarrhythmic substrate in the rat model of heart failure due to chronic volume overload. Anat Rec (Hoboken) 2011;294: 102-111.

18 Opie LH, Commerford PJ, Gersh BJ, Pfeffer MA: Controversies in ventricular remodelling. Lancet 2006;367:356-367.

19 Levy D, Garrison RJ, Savage DD, Kannel WB, Castelli WP: Prognostic implications of echocardiographically determined left ventricular mass in the Framingham Heart Study. N Engl J Med 1990;322:1561-1566.

20 Jacob R, Gulch RW: The functional significance of ventricular geometry for the transition from hypertrophy to cardiac failure. Does a critical degree of structural dilatation exist? Basic Res Cardiol 1998;93:423-429.

21 Gardner JD, Brower GL, Janicki JS: Gender differences in cardiac remodeling secondary to chronic volume overload. J Card Fail 2002; 8:101-107.

-22 Zaffran S, Kelly RG, Meilhac SM, Buckingham ME, Brown NA: Right ventricular myocardium derives from the anterior heart field. Circ Res 2004;95:261-268.
23 Banerjee D, Haddad F, Zamanian RT, Nagendran J: Right ventricular failure: a novel era of targeted therapy. Curr Heart Fail Rep 2010;7:202-211.

24 Melenovsky V, Benes J, Skaroupkova P, Sedmera D, Strnad H, Kolar M, Vlcek C, Petrak J, Benes J Jr, Papousek F, Oliyarnyk O, Kazdova L, Cervenka L: Metabolic characterization of volume overload heart failure due to aorto-caval fistula in rats. Mol Cell Biochem 2011;354:83-96.

-25 Benes J, Kazdova L, Drahota Z, Houstek J, Medrikova D, Kopecky J, Kovarova N, Vrbacky M, Sedmera D, Strnad H, Kolar M, Petrak J, Benada O, Skaroupkova P, Cervenka L, Melenovsky V: Effect of metformin therapy on cardiac function and survival in a volume-overload model of heart failure in rats. Clin Sci (Lond) 2011;121:29-41.

26 Dickstein K, Cohen-Solal A, Filippatos G, McMurray JJ, Ponikowski P, Poole-Wilson PA, Stromberg A, van Veldhuisen DJ, Atar D, Hoes AW, Keren A, Mebazaa A, Nieminen M, Priori SG, Swedberg K, Vahanian A, Camm J, De CR, Dean V, Dickstein K, Filippatos G, Funck-Brentano C, Hellemans I, Kristensen SD, McGregor K, Sechtem U, Silber S, Tendera M, Widimsky P, Zamorano JL: ESC guidelines for the diagnosis and treatment of acute and chronic heart failure 2008: the Task Force for the Diagnosis and Treatment of Acute and Chronic Heart Failure 2008 of the European Society of Cardiology. Developed in collaboration with the Heart Failure Association of the ESC (HFA) and endorsed by the European Society of Intensive Care Medicine (ESICM). Eur Heart J 2008;29:2388-2442. 